\title{
La crinopexie : un modèle décrivant les mécanismes qui régissent la biodisponibilité des facteurs de croissance
}

Les crinopexines sont des facteurs de croissance qui sont sécrétés (crino-), et adhèrent à des structures cellulaires sous une forme biologiquement inactive (-pexie). Deux familles de ces agents peuvent être distingués, selon qu'ils adhèrent à des protéoglycanes de la matrice extracellulaire (type I) ou qu'ils demeurent transmembranaires avant d'être libérés dans l'espace extracellulaire (type II). Les FGF (fibroblast growth factors) et le TGF $\beta$ (transforming growth factor $\beta$ ) sont du premier type alors qu'EGF et TGF $\alpha$ sont du second. La biodisponibilité des facteurs stockés sous une forme inactive est contrôlée par des mécanismes pouvant dépendre autant de la cellule productrice que de la cellule cible et de l'environnement. La crinopexie explique, notamment, les relations étroites entre l'activité cellulaire et l'état de la matrice extracellulaire ; elle est pour l'organisme un moyen de réaliser un niveau supérieur d'intégration de l'action des facteurs de croissance.

Jean-Jacques Feige Andrew Baird

\section{ADRESSES}

J.-J. Feige : directeur de recherche à l'Inserm. Inserm U. 244, biochimie des régulations cellulaires endocrines, département de biologie moléculaire et structurale, Centre d'études nucléaires, $85 \mathrm{X}, 38041$ Grenoble, France. A. Baird : directeur du département de biologie moléculaire et cellulaire de la croissance. Molecular and cellular growth biology, the Whittier Institute for Diabetes and Endocrinology, 9894 Genesee Avenue, La Jolla, CA 92037, USA.

$m / s n^{\circ} 8$, vol. 8 , octobre 92 es mécanismes qui régissent la prolifération et la différenciation cellulaires mettent en jeu des molécules circulantes qui agissent à distance, telles que les hormones (sécrétions endocrines), des molécules qui agissent à proximité (sécrétions paracrines) ou des facteurs qui agissent sur le lieu même de leur synthèse (sécrétions autocrines) [1, 2]. Les mécanismes régissant l'action des facteurs endocrines apparaissent très différents de ceux qui contrôlent l'activité des facteurs autocrines et paracrines. Dans les modèles de sécrétions endocrines, la synthèse et la sécrétion de la molécule trophique représentent l'étape d'initiation de la réponse biologique. En revanche, quelques observations récentes ont établi que les mécanismes qui régissent l'activité des facteurs de croissance et de différenciation sont très différents. Par exemple, ces facteurs sont souvent localisés à l'extérieur de leurs cellules cibles dans une forme qui semble être biologiquement inactive [3-8]. Dans ce cas, l'étape d'initiation semble être la libération de leurs lieux de stockage, permettant ainsi leur transfert vers des récepteurs de signalisation. Nous proposons ici d'appeler " crinopectines ", ces molécules qui sont sécrétées (crino-) par les cellules et qui adhèrent (-pectines) à des structures péricellulaires spécifiques (matrice extracellulaire, surface cellulaire, protéoglycanes...) où elles demeurent séquestrées sous une 


\section{RÉFÉRENCES}

1. Sporn MB, Todaro GJ. Autocrine secre tion and malignant transformation of cells $N$ Engl J Med 1985; 303 : 878-80.

2. Heldin $\mathrm{CH}$, Westermark B. Growth fac tors : mechanism of action and relation to oncogenes. Cell 1984; 37 : 9-20.

3. Baird A, Bohlen, P. Fibroblast growth factors. In : Sporn MB, Roberts AB, eds. Peptide Growth Factors and their Receptors - 1 New York: Springer-Verlag, 1990 : 369-403.

4. Baird A, Ling N. Fibroblast growth factors are present in the extracellular matrix produced by endothelial cells in vitro : implications for a role of heparinase-like enzymes in the neovascular response. Biochem Biophys Res Commun 1987 ; 142 : 428-35.

5. Gonzalez AM, Buscaglia M, Ong M Baird A. Distribution of basic fibroblast growth factor in the 18-day rat fetus : localization in the basement membranes of diverse tissues. I Cell Biol 1990; 110 753-65.

6. Thompson NL, Flankers KC, Smith JM, Ellingsworth LR, Roberts AB, Sporn MB. Expression of transforming growth factor- $\beta$ in specific cells and tissues of adult and neonatal mice. I Cell Biol 1989 ; 108 : 661-9. 7. Gordon MY, Riley GP, Watt SM, Greaves MF. Compartmentalization of a haematopoietic growth factor (GM-CSF) by glycosaminoglycans in the bone marrow microenvironment. Nature 1987 ; 326 : 403-5.

8. Bashkin $P$, Doctrow S, Klagsbrun $M$ Svahn CM, Folkman J, Vlodavsky I. Basic fibroblast growth factor binds to subendothelial extracellular matrix and is released by heparitinase and heparin-like molecules. Biochemistry 1989 ; 28 : 1737-43.

9. Massagué J. Transforming growth factor$\alpha$. A model for membrane-anchored growth factors. J Biol Chem 1990; 265 : 21393-6. 10. Burgess WH, Maciag T. The heparinbinding (fibroblast) growth factor family of proteins. Ann Rev Biochem 1989; 58 575-606.

11. Emoto N, Gonzalez AM, Walick PA, et al. Basic fibrobast growth factor (FGF) in the central nervous system : identification of specific loci of bFGF expression in the rat brain Growth Factors 1989 ; 2 : 21-9.

12. Florkiewicz RZ, Baird A, Gonzalez AM. Multiple forms of bFGF : differential nuclear and cell surface localization. Growth Factors $1991 ; 4$ : 265-75.

13. Brigstock DR, Sasse J, Klagsbrun M Subcellular distribution of basic fibroblas growth factor in human hepatoma cells. Growth Factors 1991; 4 : 189-96.

14. Roberts R, Gallagher J, Spooncer E, Allen TD, Blooomfield F, Dexter TM. Heparan sulphate bound growth factors : a mechanism for stromal cell mediated haemopoiesis. Nature 1988 ; 332 : 376-8.

15. Masumoto A, Yamamoto N. Sequestration of a hepatocyte growth factor in extracellular matrix in normal adult rat liver. Biochem Biophys Res Commun 1991 ; 174 : 90-5. 16. Wozney JM, Rosen V, Celeste AJ, et al. Novel regulators of bone formation: molecular clones and activities. Science 1988 $242: 1528-34$ forme latente dans l'environnement cellulaire local. Nous proposons également le terme " crinopexie " pour définir le(s) mécanisme(s) de régulation cellulaire de la biodisponibilité des crinopectines.

Selon ce concept de crinopexie, le mécanisme de déclenchement du signal biologique implique la libération du facteur trophique de ses lieux de stockage dans l'environnement local de la cellule cible. De ce fait, la libération et l'activation des crinopectines peuvent être contrôlées par la cellule cible aussi bien que par la cellule lieu de synthèse. L'étude du contrôle de la biodisponibilité des crinopectines représente un domaine d'investigation crucial pour la compréhension du mécanisme d'action de ces molécules.

\section{Caractéristiques d'une crinopectine}

Par définition, les crinopectines sont abondamment distribuées dans les tissus de l'organisme puisqu'elles sont séquestrées à l'extérieur des cellules, dans la matrice extracellulaire, dans les membranes basales ou associées avec les protéoglycanes de surface

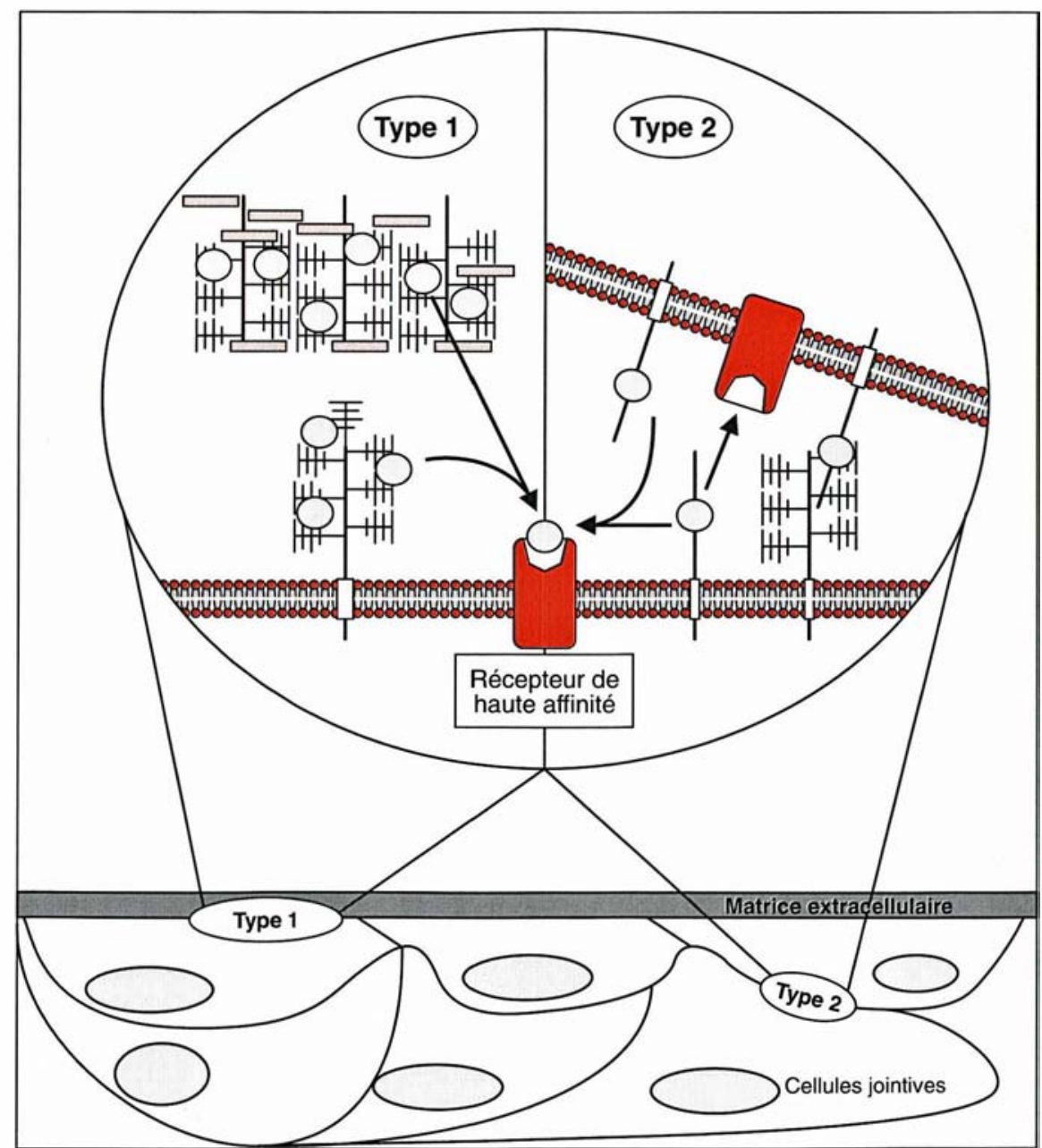

Figure 1. Les crinopectines. Les deux types de crinopectines sont représentés ici. Les crinopectines de type I sont associées à des protéoglycanes péricellulaires ou à des composants de la matrice extracellulaire. Les crinopectines de type II sont ancrées dans la membrane plasmique sous leur forme pré-

curseur, $O$; crinopectine; H+ glycosaminoglycane; _ protéine core ; $\square$, protéine de la matrice extracellulaire. 
(figure 1). La capacité de se lier à l'héparine est un phénomène caractéristique mais ne suffit pas en soi à définir une crinopectine. En effet, plusieurs protéines de liaison de l'héparine sont solubles et ne se retrouvent pas associées à la surface cellulaire (histones, FGF-5, FGF-6,...). Il existe par ailleurs plusieurs protéines qui ne se lient pas à l'héparine et qui sont tout de même des crinopectines (EGF, $\mathrm{TGF} \alpha \ldots)$. Les crinopectines peuvent être regroupées en deux classes (Tableau I).

- Les crinopectines de type I sont associées à la matrice extracellulaire ou aux composants péricellulaires (figure 1). La liaison est souvent dissociable par la force ionique tel que le $\mathrm{NaCl}(2 \mathrm{M})$ ou par un $\mathrm{pH}$ acide. - Les crinopectines de type II font partie intégrante de la membrane plasmique grâce à la présence d'un domaine transmembranaire dans la séquence peptidique de leur précurseur. Afin d'être actives, ces crinopectines doivent être libérées de leur partie précurseur par un processus enzymatique spécifique. Cependant, de nombreux exemples montrent qu'elles peuvent également agir directement sous leur forme précurseur sur les récepteurs d'une cellule cible adjacente sans être internalisées (facteurs juxtacrines) [9].

Les facteurs de croissance des fibroblastes (FGF) acide (a) et basique (b) ${ }^{*}$ sont des prototypes de crinopectines de type I. Ces facteurs présentent la propriété d'interagir très fortement avec l'héparine, les héparanesulfates, et avec la matrice extracellulaire des cellules $[4,8,10]$. Cette propriété est également observée in vivo. La localisation du bFGF montre qu'il est distribué de façon presque ubiquitaire dans les membranes basales de divers tissus périphériques alors que son ARNm est indétectable dans ces tissus $[5,11]$. Dans quelques cas, tels que les cellules en culture et le cerveau, le bFGF ne se comporte pas comme une crinopectine de type I puisqu'il est localisé à l'intérieur de la cellule, parfois même dans le noyau [5, 12, 13]. Dans ces

\footnotetext{
* $F G F$ acide $=F G F 1 ; F G F$ basique $=F G F 2$. Voir l'article de F. Coulier et al., p. 811 de ce
} numéro.

tissus, l'ARNm est détectable et la crinopexie ne semble pas être le mécanisme qui régit son activité.

Il existe plusieurs autres exemples de crinopectines de type I (Tableau I). Plusieurs études immunohistochimiques ont démontré que le facteur de transformation cellulaire $\beta$ (TGF $\beta$ ) ainsi que diverses interleukines (GMCSF, IL3) sont aussi des crinopectines de type I $[6,7,14]$. Tout récemment, la présence du facteur de croissance des hépatocytes (HGF) et des protéines de morphogenèse de l'os (BMP) a également été mise en évidence dans la matrice extracellulaire $[15,17]$. L'appartenance du facteur de croissance épidermique qui se lie à l'héparine (HB-EGF) [18] au groupe des crinopectines de type I est suggéré par sa capacité à se lier à l'héparine mais reste à démontrer. Deux exemples bien caractérisés de crinopectines de type II sont représentés par les précurseurs du facteur de croissance épidermique (EGF) et de son homologue, le facteur de transformation $\alpha(\mathrm{TGF} \alpha)$. Ces molécules existent sous forme de précur- seurs transmembranaires susceptibles soit de libérer les facteurs de croissance actifs EGF et TGF $\alpha$, soit d'interagir avec des récepteurs spécifiques situés sur des cellules cibles voisines $[19,20]$. Le facteur de nécrose des tumeurs (TNF $\alpha)$ et deux facteurs de croissance hématopoiétiques, CSF1 et le ligand du récepteur c-Kit, sont d'autres exemples de facteurs biologiquement actifs tout en étant ancrés dans la membrane plasmique et répondant aux critères des crinopectines de type II $[21,25]$ (Tableau I). Très récemment, la mise en évidence de l'activité mitogène d'une protéine de fusion de l'oncogène $v$-sis ancrée dans la membrane plasmique par le domaine transmembranaire d'une protéine virale, démontre qu'il est possible de créer des crinopectines de type II artificielles [26].

La crinopexie : un mécanisme régissant la biodisponibilité

Afin de déclencher leur(s) effet(s) bio-

\begin{tabular}{|c|c|c|c|}
\hline \multicolumn{4}{|c|}{$\begin{array}{l}\text { Tableau I } \\
\text { MPLES DE }\end{array}$} \\
\hline $\begin{array}{l}\text { Crinopectine } \\
\text { de type I }\end{array}$ & Références & $\begin{array}{l}\text { Crinopectine } \\
\text { de type II }\end{array}$ & Références \\
\hline $\begin{array}{l}\text { Facteurs de croissance } \\
\text { des fibroblastes } \\
\text { acide et basique (FGF-1 } \\
\text { et FGF-2) } \\
\text { FGF-3 (int-2) }\end{array}$ & $\begin{array}{r}{[5]} \\
{[4]} \\
{[8]} \\
{[36]}\end{array}$ & $\begin{array}{l}\text { Facteur de croissance } \\
\text { épidermique (EGF) } \\
\text { Facteur de transforma- } \\
\text { tion cellulaire } \alpha \\
\text { (TGF } \alpha \text { ) } \\
\text { Facteur de croissance } \\
\text { épidermique liant } \\
\text { I'héparine (HB-EGF) }\end{array}$ & $\begin{array}{r}{[19]} \\
{[20]} \\
{[9]} \\
{[18]}\end{array}$ \\
\hline $\begin{array}{l}\text { Facteur de transforma- } \\
\text { tion cellulaire (TGF } \beta \text { ) }\end{array}$ & $\begin{array}{r}{[34]} \\
{[6]}\end{array}$ & $\begin{array}{l}\text { Facteur de nécrose des } \\
\text { tumeurs (TNF } \alpha)\end{array}$ & [21] \\
\hline $\begin{array}{l}\text { Facteur de croissance } \\
\text { des granulocytes et des } \\
\text { macrophages-forme } 1 \\
\text { (GM-CSF) }\end{array}$ & [7] & $\begin{array}{l}\text { Facteur de croissance } \\
\text { des granulocytes et des } \\
\text { macrophages-forme } 2 \\
\text { (GM-CSF) }\end{array}$ & [43] \\
\hline Interleukine 3 & {$[14]$} & $\begin{array}{l}\text { Colony-stimulating } \\
\text { factor-1 (CSF-1) }\end{array}$ & $\begin{array}{l}{[22]} \\
{[23]}\end{array}$ \\
\hline $\begin{array}{l}\text { Facteur de croissance } \\
\text { des hépatocytes (HGF) }\end{array}$ & [15] & $\begin{array}{l}\text { Ligand de c-kit ou fac- } \\
\text { teur de croissance des } \\
\text { mastocytes }\end{array}$ & $\begin{array}{l}{[24]} \\
{[25]}\end{array}$ \\
\hline $\begin{array}{l}\text { Protéines de morphoge- } \\
\text { nèse osseuse (BMPs et } \\
\text { ostéogénine) }\end{array}$ & $\begin{array}{l}{[16]} \\
{[17]}\end{array}$ & & \\
\hline
\end{tabular}


17. Luyten FP, Cunningham NS, Ma S. Purification and partial amino acid sequence of osteogenin, a protein initiating bone differenciation. J Biol Chem 1989; 264 : 13377-80.

18. Higashiyama S, Abraham JA, Miller J, Fiddes JC, Klagsbrun M. A heparin-binding growth factor secreted by macrophage-like cells that is related to EGF. Science 1991; 251: 936-9.

19. Mroczkowski B, Reich M, Chen K, Bell GI, Cohen S. Recombinant human epidermal growth factor precursor is a glycosylated membrane protein with biological activity. Mol Cell Biol 1989 ; 9 : 2771-8.

20. Teixido J, Gilmore R, Lee DC, Massagué J. Integral membrane glycoprotein properties of the prohormone protransforming growth factor- $\alpha$. Nature 1987 ; $326: 883-5$.

21. Kriegler M, Perez C, Defay K, Albert I, Lu SD. A novel form of TNF/cachectin is a cell surface cytotoxic transmembrane protein ; ramifications for the complex physiology of TNF. Cell $1988 ; 53$ : 45-53. 22. Rettenmeier CW, Roussel MF, Ashmun RA, Ralph P, Price K, Sherr CJ. Synthesis of membrane-bound colonystimulating factor 1 (CSF-1) and transmodulation of CSF-1 receptors in NIH 3T3 cells transformed by contransfection of the human CSF-1 and c-fms (CSF-1 receptors) genes. Mol Cell Biol 1987 ; 7 : 2378-85.

23. Stein J, Borzillo GV, Rettenmier CW. Direct stimulation of cells expressing receptors for macrophage colony-stimulating factor (CSF-1) by a plasma membrane-bound precursor of human CSF-1. Blood 1990 ; 76 : 1308-14

24. Anderson DM, Lyman SD, Baird A, et al. Molecular cloning of mast cell growth factor, a hematopoietin that is active in both membrane bound and soluble forms. Cell $1990 ; 63: 235-43$

25. Flanagan JG, Leder P. The kit ligand : a cell surface molecule altered in steel mutant fibroblasts. Cell 1990 ; 63 : 185-94. 26. Lee BA, Donoghue DJ. Membraneanchored from of $v$-sis/PDGF-B induces mitogenesis without detectable PDGF receptor autophosphorylation. J Cell Biol 1991; $113: 361-70$

27. Tryggvason K, Höyhtyä M, Salo $T$. Proteolytic degradation of extracellular matrix in tumor invasion. Biochim Biophys Acta 1987 ; 907 : 191-217.

28. Sommer A, Rifkin DB. Interaction of heparin with human basic fibroblast growth factor : protection of the angiogenic protein from proteolytic degradation by a glycosaminoglycan. J Cell Phys 1989 ; 138 : 215-20. 29. Feige JJ, Baird A. Basic fibroblast growth factor is a substrate for protein phosphorylation and is phosphorylated by capillary endothelial cells in culture. Proc Natl Acad Sci USA 1989 ; 86 : 3174-8.

30. Vilgrain I, Baird A. Phosphorylation of basic fibroblast growth factor by a protein kinase associated with the outer surface of a target cell. Mol Endocrinol 1991; 5 : 1003-12.

chromatography of fibroblast growth factors. J Biol Chem 1988 ; 263 : 9059-62.

32. Pettman B, Janet T, Labourdette G Sensenbrenner M, Manthrope M, Varon S. Biologically active basic fibroblast growth factor migrates at $27 \mathrm{kD}$ in non-denaturing SDS-polyacrylamide gel electrophoresis Growth Factors 1991 ; 5 : 345-52.

33. Pandiella A, Massagué J. Cleavage of the membrane precursor for transforming growth factor- $\alpha$ is a regulated process. Proc Natl Acad Sci USA 1991; 88 : 1726-30.

34. Massagué J. The transforming growth factor- $\beta$ family. Ann Rev Cell Biol 1990; 6 : 597-640.

35. Wang XF, Lin $\mathrm{H}, \mathrm{Ng}$-Eaton E, Downward J, Lodish HF, Weinberg RA. Expression cloning and characterization of the TGF $\beta$ type III receptor. Cell 1992; 67 : 797-805.

36. Kief er MC, Stephans JC, Crawford K, Okino K, Barr PJ. Ligand affinity cloning and structure of a heparan sulfate proteoglycan that binds basic fibroblast growth factor. Proc Natl Acad Sci USA 1990; 87 : 6985-9.

37. Yayon A, Klagsbrun M, Esko JD, Leder P, Ornitz DM. Cell surface, heparinlike molecules are required for binding of basic fibroblast growth factor to its high affinity receptor. Cell $1991 ; 64$ : 841-8.

38. Rapraeger AC, Krufka A, Olwin BB. Requirement of heparan sulfate for bFGFmediated fibroblast growth and myoblast differentiation. Science $1991 ; 252$ : 1705-8. 39. Klagsbrun M, Baird A. A dual receptor system is required for basic fibroblast growth factor activity. Cell 1991; 67 : 229-31

40. Lee PL, Johnson DE, Cousens LS, Fried VA, Williams LT. Purification and complementary DNA cloning of a receptor for basic fibroblast growth factor. Science 1989 ; 245 : 57-60.

41. Gonzalez AM, Buscaglia ML Fuller J, Dahl R, Carman L, Baird A. Local fate and distribution of locally infused bFGF : the example of rat brain and the xenopus tail mesenchyme. Ann NY Acad Sci 1991; 638 : 416-9.

42. Higashiyama S, Abraham JA, Miller J, Fiddes JC, Klagsbrun M. A heparin-binding growth factor secreted by macrophage-like cells that is related to EGF. Science 1991; 251: 936-9.

43. Gough NM, Metcalf D, Gough J, Grail D, Dunn AR. Structure and expression of the mRNA for murine granulocytemacrophage colony stimulating factor. EMBO J 1985 ; 4 : 645-53.

44. Kief er $P$, Peters $G$, Dickson C. The INT-2/FGF-3 oncogene product is secreted and associates with the extracellular matrix : implications for cell transformation. Mol Cell Biol 1991 ; 11 : 5929-36.

45. Mascarelli F, Raulais D, Courtois Y. Fibroblast growth factor phosphorylation and receptors in rod outer segments. $E M B O J$ $1989 ; 8: 2265-73$

46. Assouline $M$, Hutchinson $C$, Morton $\mathrm{K}$, et al. In vivo binding of topically applied human bFGF on rabbit corneal epithelial wound. Growth Factors 1989; 1 : 251-61. logique(s), les crinopectines stockées à la surface cellulaire doivent être rendues disponibles pour leurs récepteurs de signalisation. Les mécanismes moléculaires qui régissent la biodisponibilité des crinopectines sont encore en grande partie inconnus. Nous développerons ici à titre d'exemple ceux que nous envisageons pour le bFGF (figure 2).

La matrice extracellulaire et les protéoglycanes qui lient le bFGF sont sujets à une dégradation protéolytique et glycolytique par des enzymes telles que la plasmine, les cathepsines, les collagénases, la stromélysine ou diverses protéoglycanases. Physiologiquement, divers inhibiteurs sécrétés par les cellules (inhibiteurs de l'activateur du plasminogène, $\alpha_{2}$-macroglobuline, protéase nexines) se comportent en antagonistes de ces enzymes [27]. Tout dérèglement de cet équilibre dans le sens d'une activité protéolytique accrue peut libérer le bFGF de son compartiment de séquestration péricellulaire. Il peut alors être présenté à son récepteur de haute affinité afin de déclencher le signal mitogène. Complexé à des fragments d'héparane sulfate, le FGF est stabilisé et n'est ainsi pas sujet à la protéolyse [28].

La possibilité qu'il existe des modifications post-traductionnelles des crinopectines entraînant un changement de leur affinité pour leur récepteur de séquestration ou pour leur récepteur de signalisation, représente un autre mécanisme de régulation de leur biodisponibilité. Dans le cas du FGF, ces modifications peuvent inclure sa phosphorylation [29, 30, 45], la formation de complexes avec le cuivre [31] et la formation de dimères [32].

A l'équilibre, les crinopectines telles que les FGF acide et basique sont distribuées entre des sites de liaison de basse affinité représentés pas les récepteurs de stockage/livraison et des sites de forte affinité que sont les récepteurs de signalisation à activité protéine-tyrosine kinase $[3,10]$. On peut donc prédire qu'un effecteur cellulaire capable d'augmenter le nombre de récepteurs de haute affinité pourrait, en principe, provoquer le déplacement de FGF de ses sites de stockage vers ses récepteurs de signalisation. Cette variation du nombre 


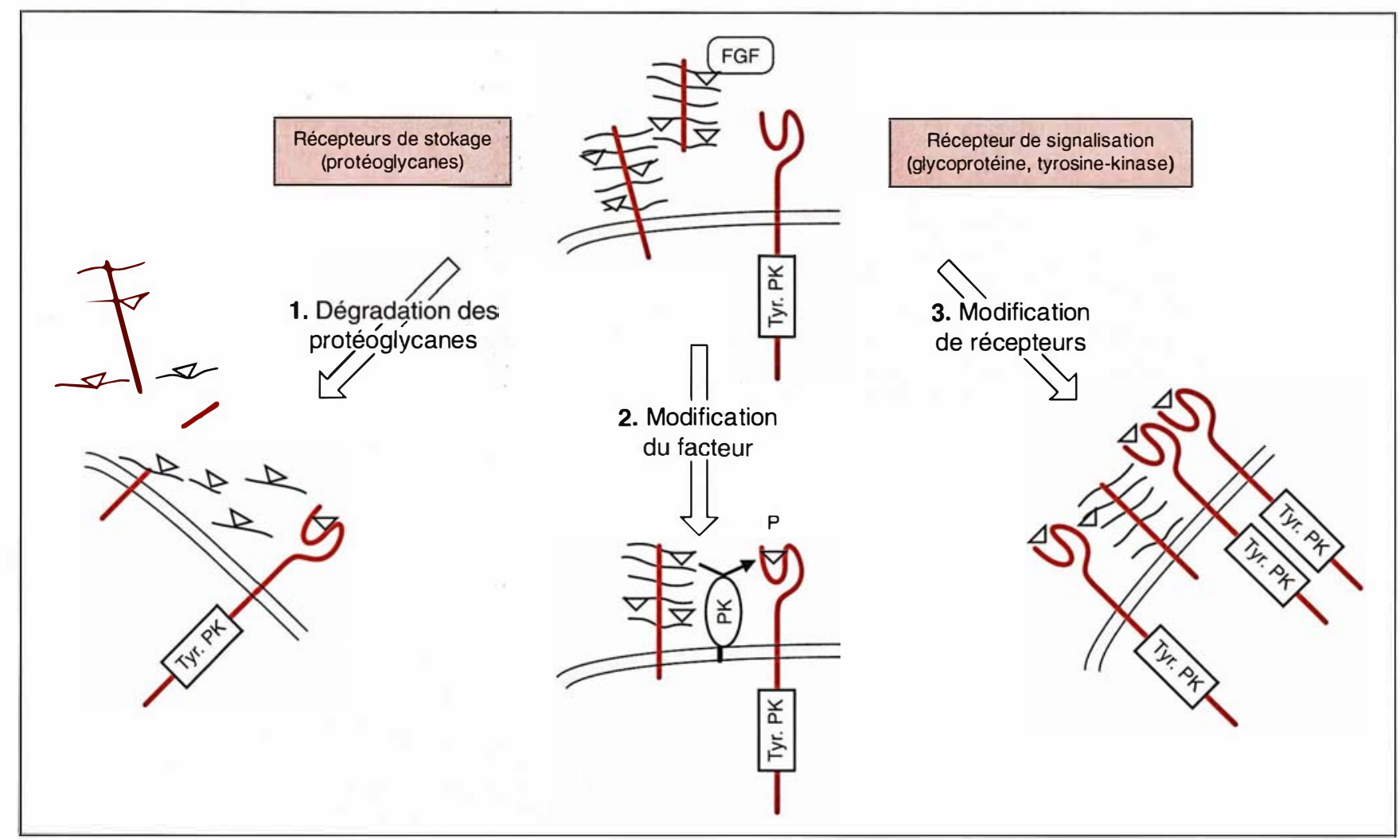

Figure 2. Régulation de la biodisponibilité des crinopectines. Trois mécanismes de régulation de la disponibilité de bFGF pour ses récepteurs de haute affinité sont représentés ici. 1. La dégradation des protéoglycanes péricellulaires ou extracellulaires par des protéases ou des glycanases libère du FGF complexé à des fragments de glycosaminoglycanes, qui peut alors se lier à ses récepteurs de signalisation doués d'activité protéine-tyrosine kinase. 2. La modification post-traductionnelle du FGF (par exemple sa phosphorylation) augmente son affinité pour ses récepteurs de signalisation. 3. L'augmentation du nombre de récepteurs de haute affinité sous l'action de peptides régulateurs (hormones, facteurs de croissance...) déplace le FGF de ses sites de stockage vers ses récepteurs de signalisation. $P K$ : protéine kinase; Tyr-PK : protéine-tyrosine kinase.

de récepteurs suffirait à déclencher les signaux intracellulaires induits par la crinopectine. C'est donc la cellule cible elle-même qui, en répondant aux changements homéostatiques de son environnement, prendrait en charge la libération de ces facteurs de croissance et déclencherait leur activité biologique.

Quant aux crinopectines de type II (dont le précurseur possède un domaine peptidique transmembranaire), leur activité est probablement dépendante d'enzymes spécifiques (protéases) qui seraient capables de libérer le facteur de croissance actif. Récemment cependant, des formes du précurseur de TGF $\alpha$ ont été synthétisées qui ne sont plus substrats pour cette protéolyse mais qui sont tou jours biologiquement actives [33]. Il est par conséquent important $\mathrm{m} / \mathrm{s} n^{\circ} 8$, vol. 8, octobre 92 d'envisager la possibilité que le précurseur lui-même soit capable de stimuler la cellule dont il est issu, ou une cellule voisine avec laquelle il interagit sans quitter son lieu de synthèse. Dans ce cas, ce facteur aurait une activité autocrine ou juxtacrine.

La liaison des crinopectines de type I à la surface cellulaire ou à la matrice se fait souvent par l'intermédiaire de protéoglycanes. La caractérisation des récepteurs de $\operatorname{TGF} \beta$ a mis en évidence trois types majeurs de récepteurs dont l'un (récepteur de type III/ $\beta$-glycane) est un protéoglycane contenant des chaînes héparane sulfates et chondroïtine sulfates [34]. Cette molécule qui possède un très court domaine intracellulaire [35] représente probablement le récepteur de séquestration des TGF $\beta$. Une situation similaire existe pour le récepteur de basse affinité du bFGF dont l'identité avec le syndécane a été établie [36]. Cette molécule, qui remplit probablement une fonction de séquestration, peut aussi servir à présenter le bFGF sous une conformation adéquate à son récepteur de haute affinité [37, 39]. Ce dernier est une protéine transmembranaire qui possède une activité tyrosine kinase et transmet à la cellule cible le signal biologique des FGF [40].

\section{Conséquences physiologiques de la crinopexie}

La notion de crinopexie suggère que les facteurs de croissance demeurent à la disposition des cellules cibles sous une forme de stockage. On peut donc 
prédire que leur demi-vie in vivo est relativement longue. De fait, plusieurs groupes ont montré que l'interaction de bFGF avec les héparane sulfates le protège d'une protéolyse [28], que le bFGF injecté directement dans les tissus mésodermiques présente une longue demivie [41] ou que le bFGF lié à la membrane basale de l'épithélium cornéen est stable pendant plusieurs semaines et ne diffuse pas dans l'espace intraoculaire [46]. La conséquence physiologique de cette stabilité in vivo et de sa présence sous forme inactive dans la matrice extracellulaire est que son activité est réglée par la combinaison de sa biodisponibilité et de la capacité de la cellule cible de l'activer. De ce fait, on peut prédire que, quand la matrice extracellulaire est modifiée, l'activité des facteurs de croissance change. A ce titre, il est intéressant de noter que la modification de la matrice observée dans diverses situations physiologiques et pathophysiologiques - telles que les blessures, le diabète, les tumeurs ou même à la suite de traitements hormonaux est souvent associée à des changements de la croissance cellulaire.

\section{Conclusion}

Le concept de crinopexie et la notion de crinopectine ont été développés pour comprendre ces familles de molécules qui sont à la fois sécrétées et ancrées dans les environs de la surface cellulaire. Il est fort probable que les prochaines années verront s'élucider les mécanismes qui gouvernent leur biodisponibilité. La découverte des facteurs endocrines et hypothalamiques a longtemps soutenu le concept selon lequel la sécrétion de la molécule trophique est l'étape d'initiation de la réponse biologique. La caractérisation des crinopectines telles que les facteurs de croissance a remis en question toutes ces notions. En identifiant l'étape d'initiation qui permet le transfert des crinopectines des récepteurs de stockage vers les récepteurs de signalisation, il sera peut-être plus facile de reconnaître les mécanimes moléculaires qui régissent la croissance physiologique et pathophysiologique des cellules in vivo

\section{Summary}

The pexicrine pathway: a model for the regulation of growth factor bioavailability

Many growth factors are localized outside their target cells in what is presumed to be a biologically inactive form. Because they adhere (pexi-) to specific structures in the local environnement (matrix, cell surface, proteoglycans...), after they are secreted (crinc) by cells and appear to be biologically inert, we describe this phenomenon as crinopexy. This "pexicrine " pathway has led to the development of a model to describe the regulation of numerous autocrine and paracrine factors and predicts the existence of spccific signals responsible for their activation. These include an enzymatic release from the matrix stores, post-translational modification and changes in high and low affinity receptor expression. In this review, we fully describe this pexicrine pathway, define the criteria a molecule must meet to be considered a "crinopexin ", present how these molecules might be regulated and discuss the physiological significance of the model.

\section{Remerciements}

Nous sommes reconnaissants au professeur Roger Guillemin d'avoir été l'inspirateur de la notion de crinopexie, sur la base des expériences de son laboratoire auxquelles nous et nos collègues avons participé, et de nous avoir permis, à travers de nombreuses discussions scien tifiques, de développer ce nouveau concept. Nous remercions Denise Higgins et Sonia Lidy pour leur aide éditoriale. Nous dédions cet article à nos mères respectives pour leur amour de la languc française.

\section{TIRÉS A PART}

J.J. Feige. 